\title{
Generation of programmable 3D optical vortex structures through devil's vortex-lens arrays
}

\author{
Alejandro Calabuig, ${ }^{1,2}$ Sergio Sánchez-Ruiz, ${ }^{1,2}$ Lluís Martínez-León, ${ }^{1,2, *}$ \\ Enrique Tajahuerce, ${ }^{1,2}$ Mercedes Fernández-Alonso, ${ }^{1,2}$ Walter D. Furlan, ${ }^{3}$ \\ Juan A. Monsoriu, ${ }^{4}$ and Amparo Pons-Marti ${ }^{3}$ \\ ${ }^{1}$ INIT—Institut de Noves Tecnologies de la Imatge, Universitat Jaume I, Castelló, Spain \\ ${ }^{2}$ GROC.UJI—Grup de Recerca d'Òptica, Dept. de Física, Universitat Jaume I, Castelló, Spain \\ ${ }^{3}$ Department d'Òptica, Universitat de València, Burjassot (València), Spain \\ ${ }^{4}$ Centro de Tecnologías Físicas, Universitat Politècnica de València, València, Spain \\ *Corresponding author: Iluis.martinez@uji.es
}

Received 28 May 2013; revised 16 July 2013; accepted 17 July 2013; posted 17 July 2013 (Doc. ID 191324); published 8 August 2013

\begin{abstract}
Different spatial distributions of optical vortices have been generated and characterized by implementing arrays of devil's vortex lenses in a reconfigurable spatial light modulator. A simple design procedure assigns the preferred position and topological charge value to each vortex in the structure, tuning the desired angular momentum. Distributions with charges and momenta of the opposite sign have been experimentally demonstrated. The angular velocity exhibited by the phase distribution around the focal plane has been visualized, showing an excellent agreement with the simulations. The practical limits of the method, with interest for applications involving particle transfer and manipulation, have been evaluated. (C) 2013 Optical Society of America

OCIS codes: $\quad$ (050.1965) Diffractive lenses; (050.1970) Diffractive optics; (050.4865) Optical vortices. http://dx.doi.org/10.1364/AO.52.005822
\end{abstract}

\section{Introduction}

As is well known, optical vortices have good performances for optical trapping because they are capable not only to trap but also to set the microparticles into rotation $[1,2]$ due to its inherent orbital angular momentum $[3,4]$.

One common method to generate optical vortices is by the use of spiral phase plates [5]. It has been demonstrated that spiral phase plates can be combined with fractal zone plates (FraZPs) [6] to produce a sequence of focused optical vortices along the propagation direction [7]. Among FraZPs, devil's vortex lenses (DVLs) deserve particular interest. Their experimental generation and characterization has

$1559-128 \mathrm{X} / 13 / 235822-08 \$ 15.00 / 0$

(C) 2013 Optical Society of America been recently reported [8-10]. It has been shown that these elements have high diffraction efficiency and that it is possible to take advantage of their particular volumetric focal structure to design versatile and efficient optical tweezers.

The interest in the generation of parallel vortex distribution has recently increased, and several new methods have been proposed to generate $2 \mathrm{D}$ and $3 \mathrm{D}$ spatial distributions of vortices. This type of light distribution can be obtained by means of interferometric techniques such as the use of modified Michelson or Mach-Zehnder interferometers [11]. Dammann gratings structures have also been employed to produce sets of parallel vortex patterns $[12,13]$. In [14] the authors describe a setup based on a Dammann vortex grating to generate a $3 \mathrm{D}$ array of focused vortices with tunable topological charge. The phase modulation of an annular aperture array 
has also been proposed [15] to create distributions of high-order vortex cones. Arrays of optical vortices have a wide range of applications in many different fields including the study of the optical angular momentum of light beams [16], micro-optomechanical pumps [17], or quantum information processing [18].

Some recent approaches for generating 3D optical structures have been based on addressing either FraZPs arrays [19] or computer-generated holograms (CGHs) [20] to a spatial light modulator (SLM). In these applications, the use of CGHs [21], instead of displaying a direct pattern of lens arrays, avoids certain restrictions (for instance, the constraints related to the resolution of any diffractive element on the display). Thus, a vortex structure can be implemented through multiplexed holograms in the Fourier domain, with optical carriers of different periods. However, this approach presents other technical limitations concerning the hologram codification, like the limited-phase range available. Moreover, an iterative algorithm for generating the CGH is needed to improve the reconstruction quality [20].

In this paper, we propose a simple method to obtain elaborate spatial distributions of vortices using an array of DVLs generated in a reconfigurable SLM. The use of an SLM allows us the possibility to change in a simple way the characteristics of individual lenses, such as their focal length or their topological charge in order to obtain different and versatile configurations. Different sets of simulations and experimental results demonstrating the implementation of compound 3D optical vortex structures by means of an array of DVLs are presented. We include an experimental verification of how the compound phase distribution rotates as it propagates. In addition, an evaluation of the restrictions for the practical implementation of these arrays of vortices is reported.

The remaining of the paper is as follows. In Section 2 , the basic theory for generating a DVL is revised. The experimental implementation of different arrays of DVLs generating a compound 3D configuration of optical vortices is described in Section $\underline{3}$, whereas in Section 4 experimental results are presented in fine agreement with the simulations performed. In addition, Section 4 includes the visualization of the angular velocity exhibited by the phase distribution as it propagates and the study of the experimental limits and restrictions of the approach. Finally, Section 5 summarizes the main conclusions of this work.

\section{Basic Theory}

A devil's lens (DL) is a rotationally symmetric diffractive lens whose phase profile is designed from a devil's staircase function [22]. The triadic Cantor set [23] is often chosen as the devil's staircase function to carry out the generation of the DLs. This set is constructed as follows: first of all a straight-line segment of unit length is defined (stage $s=0$ ); then this segment is divided into three equal parts (first fractal order $s=1$ ), and the central part is removed (it becomes a disjoint gap); for the next stages (fractal orders $s=2,3,4, \ldots$ ), each segment generated in the previous stage is divided into three equal parts, the central one being removed. At stage $s$, there are $N^{s}$ segments of limits $\left[p_{s, l} q_{s, l}\right]$ with $l=1, \ldots, N^{s}-1$. Mathematically the Cantor function, or devil's staircase, is defined in the domain $[0,1]$ as

$$
F_{s}(x)=\left\{\begin{array}{cc}
\frac{l}{2^{s}} & \text { if } p_{s, l} \leq x \leq q_{s, l} \\
\frac{1}{2^{s}} \frac{x-q_{s, l}}{p_{s, l+1}-q_{s, l}}+\frac{l}{2^{s}} & \text { if } q_{s, l} \leq x \leq p_{s, l+1}
\end{array},\right.
$$

where $F_{s}(0)=0$ and $F_{s}(1)=1$. In Fig. 1 the triadic Cantor set developed up to $s=2$ and the corresponding Cantor function $F_{2}(x)$ are depicted. In this example, the Cantor set has $2^{s}=4$ segments of length $3^{-s}=1 / 9$ and $2^{s}-1=3$ gaps located at the intervals $[1 / 9,2 / 9],[3 / 9,6 / 9]$, and $[7 / 9,8 / 9]$, where $F_{2}(x)$ takes the constant values $1 / 4,2 / 4$, and $3 / 4$, respectively, and increases linearly between these intervals.

From a particular Cantor function $F_{s}(x)$, a DL is a pure-phase diffractive optical element whose transmittance is defined by

$$
Q(\zeta)=\exp \left[-i 2^{s+1} \pi F_{s}(\zeta)\right],
$$

where $\zeta=r^{2} / a^{2}$ is the normalized quadratic radial variable, and $a$ is the lens radius. As DLs are generated as circular objects, the straight line of unit length mentioned in the Cantor set corresponds to the normalized radius. We should consider an $r^{2} / a^{2}$ space because the lengths in the Cantor set are normalized, and diffractive Fresnel zone plates are conceived as objects with quadratic radial phase dependence. Using these tools, a DL with fractal order $s$ presents $2^{s}$ segments of length $3^{-s}$ and $2^{s}-1$ disjoint gaps of variable length as the corresponding Cantor function.

A DVL $[8, \underline{9}, 24]$ can be constructed from a DL by simply adding the phase variation $m \theta$, where $m$, the topological charge, is an integer and $\theta$ is the azimuthal angle. This azimuthal phase variation is introduced both in the segments and in the disjoint gaps and becomes faster as $m$ grows. Thus the transmittance of a DVL can be expressed as

$$
Q(\zeta, \theta)=\exp \left[-i 2^{s+1} \pi F_{s}(\zeta)\right] \times \exp [i m \theta]
$$

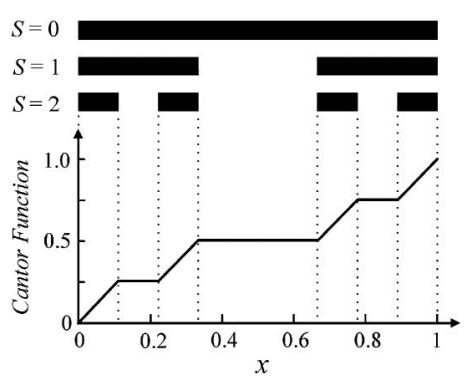

Fig. 1. Triadic Cantor set for $s=1$ and $s=2$. The Cantor function of devil's staircase for $s=2$ is plotted under the Cantor set. 


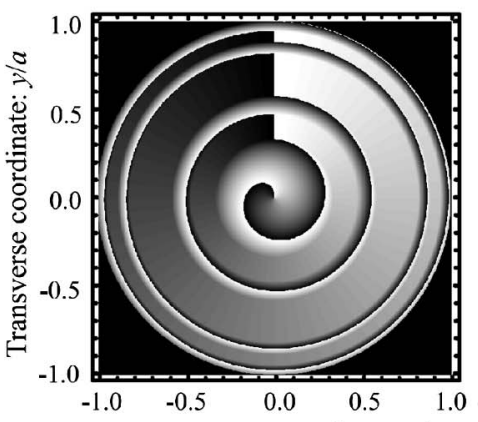

(a) Transverse coordinate: $x / a$

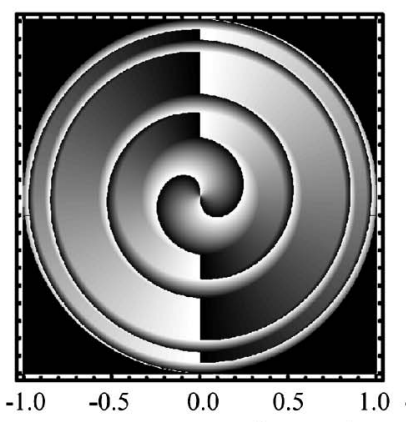

(b) Transverse coordinate: $x / a$

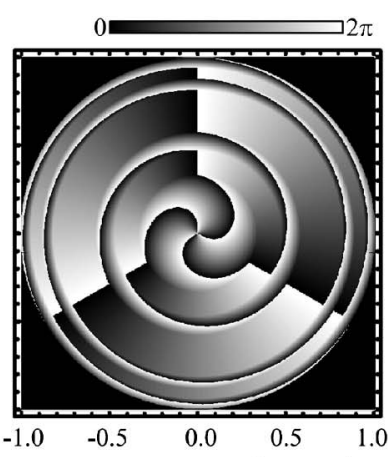

(c) Transverse coordinate: $x / a$

Fig. 2. Phase profiles for a DVL with $s=2$ and topological charge: (a) $m=1$, (b) $m=2$, and (c) $m=3$.

In this equation, the first factor, associated with a DL, has only a radial dependence with a fractal structure along the squared radial coordinate, and the second one, corresponding to a vortex lens, has a linear phase dependence on the azimuthal angle. In Fig. 2 , we have represented three DVLs with the same fractal order, $s=2$, and different values of the topological charge $m=1,2,3$. The gray levels in this figure show the continuous phase variation.

The axial behavior of these elements has been studied (see Refs. $[8, \underline{9}, 24]$ ), and the diffracted field at a given point $(z, \bar{r}, \bar{\theta})$ is characterized by the irradiance and the phase functions, which are defined, respectively, by

$I(z, r)=\left(\frac{2 \pi}{\lambda z}\right)^{2}\left|\int_{0}^{1} Q(\zeta) \exp \left[-i \frac{\pi}{\lambda z} a^{2} \zeta\right] J_{m}\left(\frac{2 \pi}{\lambda z} a r \sqrt{\zeta}\right) \mathrm{d} \zeta\right|$

$$
\Phi(z, r, \theta)=m\left(\theta+\frac{\pi}{2}\right)-\frac{2 \pi}{\lambda} z-\frac{\pi r^{2}}{\lambda z}-\frac{\pi}{2}
$$

From these equations, it is well established that a DVL has a principal focus at $f_{s}=a^{2} /\left(2 \lambda 3^{s}\right)$ and a number of subsidiary focal points surrounding it, a focal volume with a characteristic fractal profile. Each focus is an optical vortex, and a chain of doughnut-shaped foci is generated whose diameter increases with the topological charge. Additionally, the phase evolves rotating along the axial coordinate and obviously the sense of rotation depends on the sign of the topological charge.

\section{Experiment}

The experimental setup, shown in Fig. 3, employs a collimated input beam from a polarized $\overline{\mathrm{He}}-\mathrm{Ne}$ laser $(\lambda=632.8 \mathrm{~nm})$. A reconfigurable SLM displays the phase pattern representing the DVL matrices. The SLM used is a Holoeye PLUTO, with $1080 \times 1920$ square pixels of side $8 \mu \mathrm{m}$, and a gray-level codification range of 8 bits. A telescopic system after the SLM, with lenses $\mathrm{L}_{1}$ and $\mathrm{L}_{2}$, of focal lengths $f_{1}=$ $200 \mathrm{~mm}$ and $f_{2}=100 \mathrm{~mm}$ respectively, provides a magnification $M=0.5$. As reported in one of our

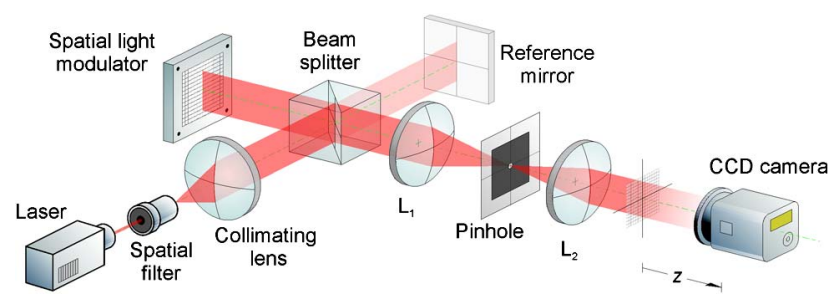

Fig. 3. Experimental setup.

previous papers [9], by implementing a linear carrier phase in the SLM, the first diffraction-order term can be isolated in the Fourier plane of the first relay lens. For that purpose, a blazed grating with a period of four pixels is added to the area covered by each lens function. Any noise due to undiffracted light, in particular to the zeroth-order diffraction, is avoided by using a pinhole to select the light corresponding to the first diffraction-order and to filter the others. Finally, a CCD camera $(1038 \times 1388$ pixels, pixel pitch of $6.45 \mu \mathrm{m}, 16$ bit gray-levels), with the help of a motorized stage, records the irradiance patterns around the main focal point of the DVLs imaged through the telescope. The reference mirror appearing as a dimmed object in Fig. $\underline{3}$ is employed just in the part of the experiment corresponding to the visualization of the orbital angular momentum, as explained below.

\section{Results and Discussion}

\section{A. Simulations and Experimental Results}

Our results demonstrate the experimental implementation of DVL matrices with programmable SLMs. Numerical simulations, according to Refs. [8,24], have been developed to compare theory and experiments. As proof of the concept, we present here the simulations and the experimental results corresponding to two matrices, though other examples have been tested.

On the one hand, a $1 \times 3$ matrix has been implemented, containing two lenses with fractal order $s=2$, and topological charge $m=2$ in both sides, and a lens with $s=2, m=-2$, in the center. This phase pattern is shown in Fig. $\underline{4(\mathrm{a})}$. 


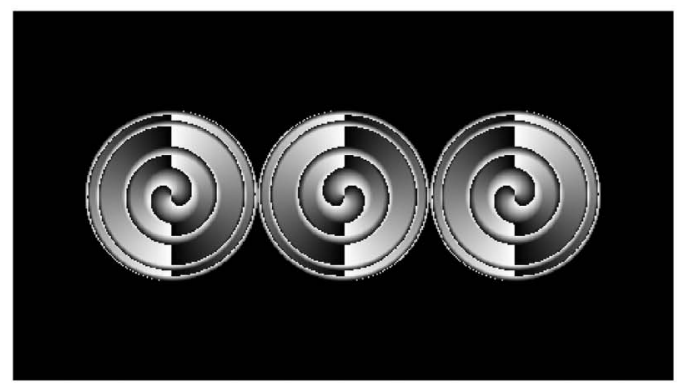

(a)

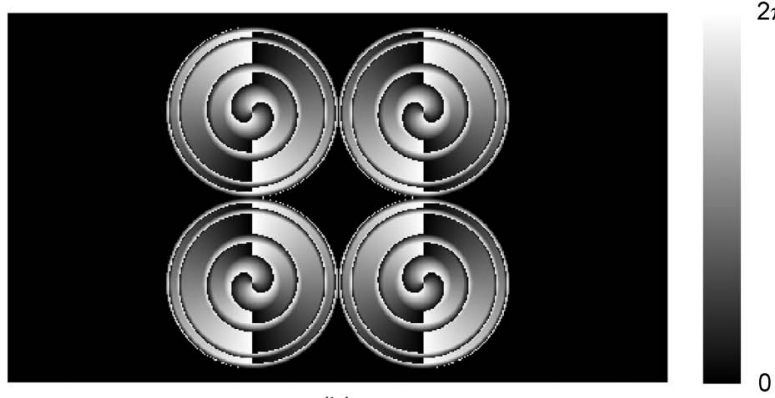

(b)

Fig. 4. Phase images of (a) the $1 \times 3$ matrix and (b) the $2 \times 2$ matrix codified in the SLM, with a DVL diameter of 499 pixels.

For each matrix, a set of images has been recorded, and the axial profile around the main focus has been evaluated. As the DVLs focal length, $f_{s}=a^{2} /\left(2 \lambda 3^{s}\right)$, depends on the lens radius, the fractal order, and the wavelength used, the main focus position is the same for all the lenses in the matrices. In Fig. 5 , simulated (a) and experimental (b) axial profiles of the $1 \times 3$ matrix described above are compared. It is worth remembering that the magnification $M$ should be taken into account when contrasting measured and
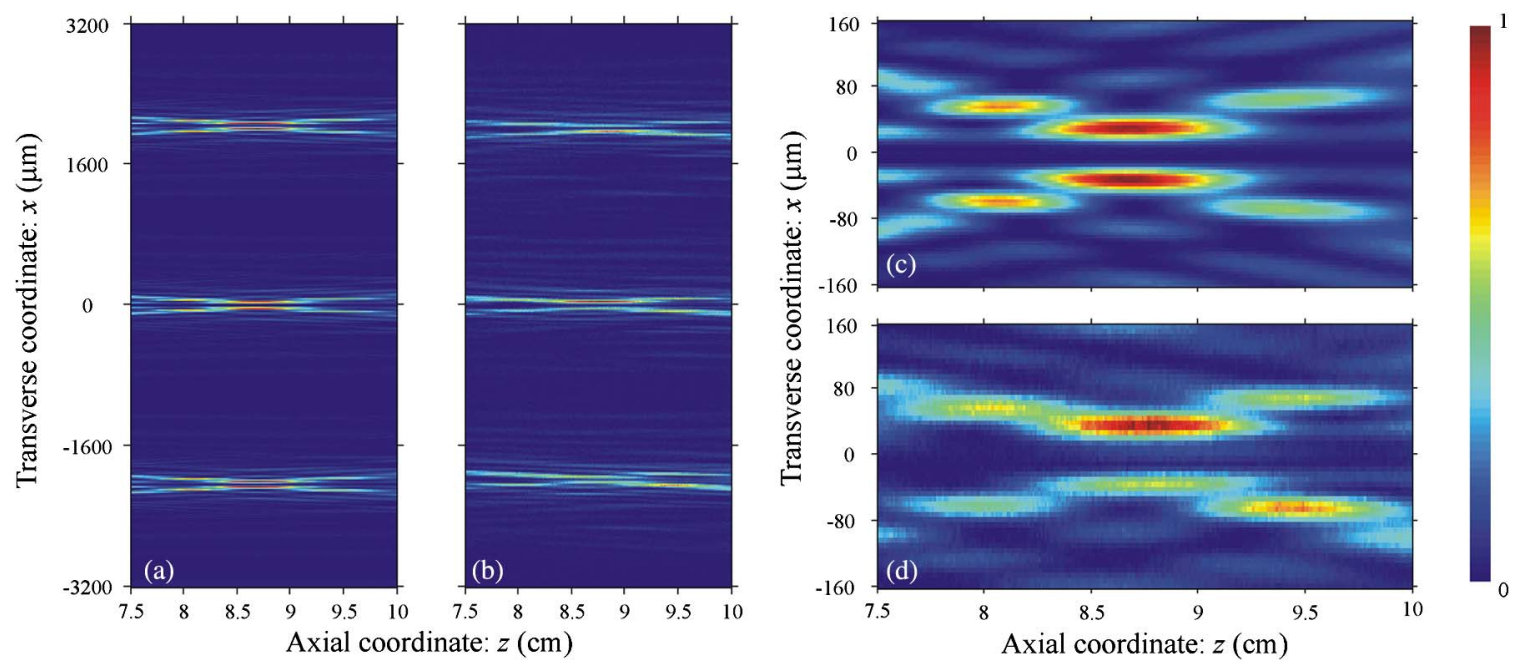

Fig. 5. Results for the $1 \times 3$ DVL matrix. Simulated (a) and experimental (b) axial profiles, and magnification for the central lens, simulation (c) and experiment (d).
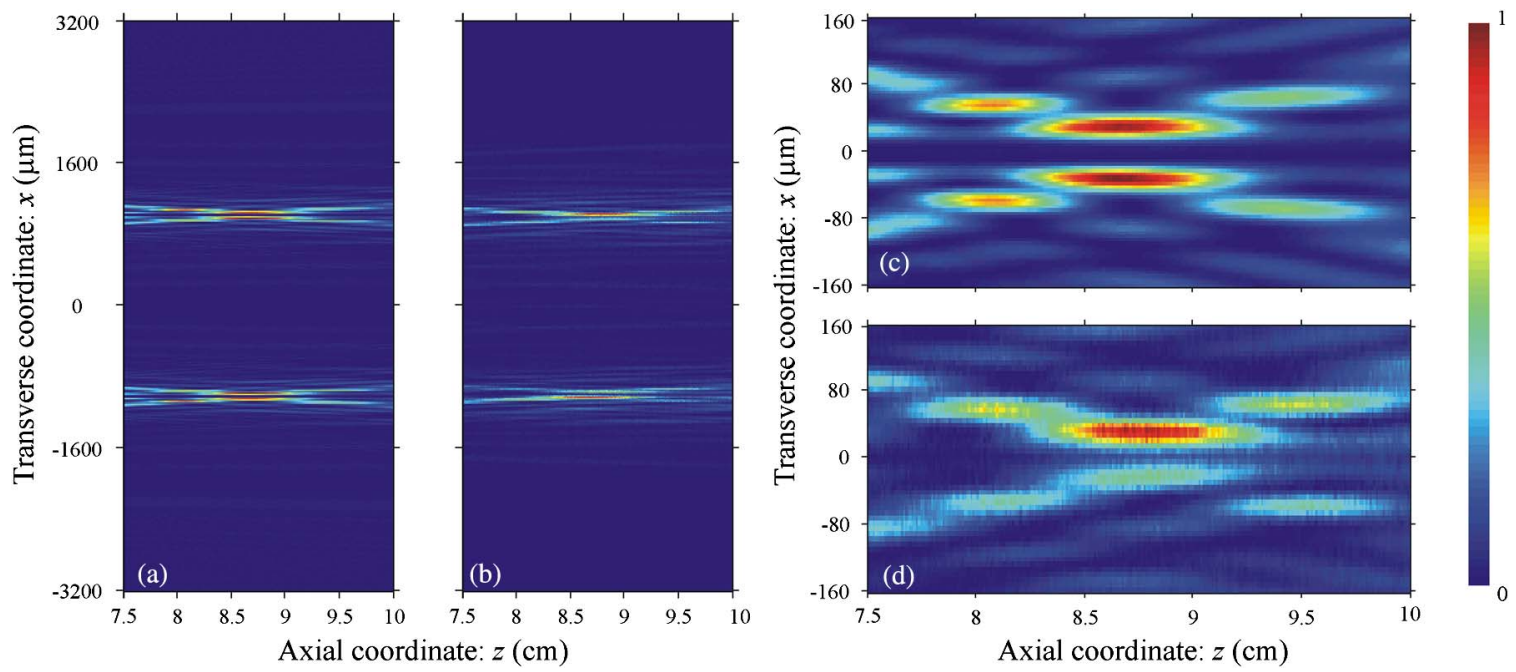

Fig. 6. Results for the $2 \times 2$ DVL matrix. Simulated (a) and experimental (b) axial profiles for the upper lenses, and magnification for the upper right lens, simulation (c) and experiment (d). 
theoretical focus positions. The representations of the axial profiles are magnified for the central lens $(s=2, m=-2)$ in Figs. 5(c) and 5(d), where the fractal nature of DVLs is clearly observed. Indeed, along the focal volume generated by the DVLs, the main focus and other subsidiary ones can be observed. A good agreement between simulated and experimental results is observed for both views. The optical aberrations, the nonideal behavior of the SLM, and the filtering process in the optical setup could be blamed for a slight discrepancy between the simulated and experimental intensity values.

On the other hand, a $2 \times 2$ matrix, with $s=2$, $m=-2$ in the main diagonal and $s=2, m=2$ elsewhere, has been studied [see Fig. 4(b)]. The simulated and experimental results are shown in Fig. $\underline{6}$. As for the $1 \times 3$ matrix, there is a satisfactory agreement between simulation and experiment. Please note that the origin for the transverse coordinate axis for Figs. 6(a) and 6(b) is different from that of Figs. $\underline{6(\mathrm{c})}$ and $\underline{6(\mathrm{~d})}$, as, in the latter, origin has been centered in the lens axis.

A series of images of the transversal planes around the main focus for the $1 \times 3$ matrix can be visualized in animated Fig. 7(a). Both simulated and experimental images are shown. In the video, the vortex effect of the DVLs can be appreciated. Three consecutive doughnuts are visualized, the second one corresponding to the main focus. The same kind of images for the $2 \times 2$ matrix can be visualized in Fig. 7(b). In Fig. 7 , the transversal distance between the centers of axial profiles is about $2 \mathrm{~mm}$. The videos cover an axial distance of $2.5 \mathrm{~cm}$ around the main focal point $(1.25 \mathrm{~cm}$ ahead and $1.25 \mathrm{~cm}$ behind the main focus).

As a method for visualizing the angular velocity exhibited by the phase distribution as it propagates, we have recorded the interference pattern between our beam and a reference one. For this part of the experiment, we take advantage of the beam splitter in front of the SLM and build a sort of Michelson interferometer using a new mirror. As a result, two colinear beams reach the CCD sensor, the one coming from the SLM and a reference beam. The reference being a plane wave, the interference pattern will provide a method for visualizing an image of the phase, in which the phase rotation is clearly observed. In animated Fig. 7(c), the simulated and experimental interference images for matrix $1 \times 3$ are shown. The counterclockwise rotation corresponds to the two side lenses, with positive topological charge $m$, and the clockwise rotation to the central lens, with a negative value for $m$. The video illustrates the capability of DVLs matrices to show a coordinated and complex angular velocity behavior, which could be of interest for particle manipulation applications.

\section{B. Experimental Limits and Restrictions}

To check the experimental restrictions of our scheme, we have theoretically and experimentally studied
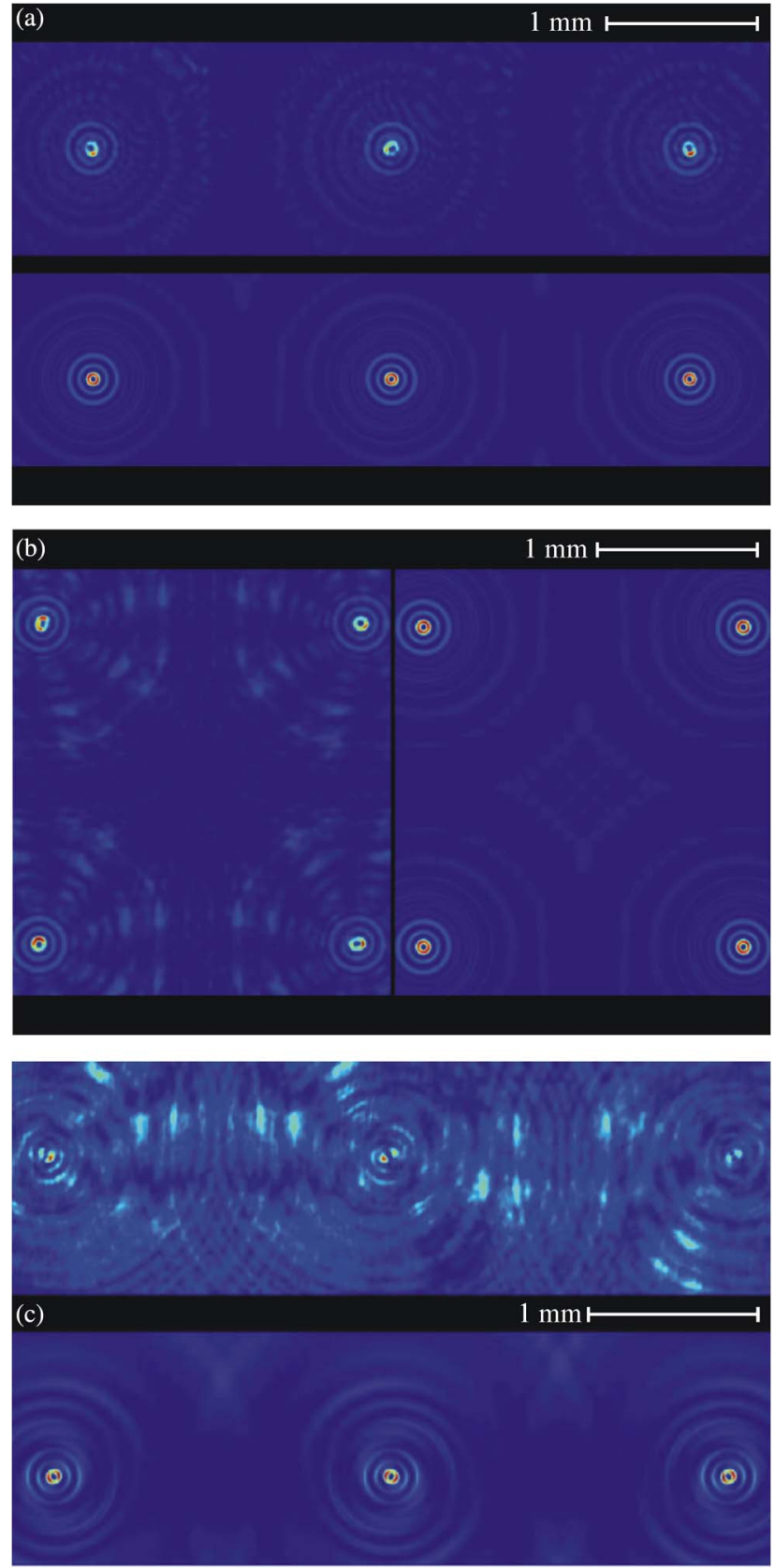

Fig. 7. Single-frame excerpts from videos showing the transversal images around the main focus. (a) $1 \times 3$ matrix: experiment (above) and simulation (below) (see Media 1). (b) $2 \times 2$ matrix: experiment (right) and simulation (left) (see Media 2). (c) Phase variation for the $1 \times 3$ matrix: experiment (above) and simulation (below) (see Media 3).

the minimum lens size and the adequate diaphragm aperture in the Fourier plane of the telescopic system. These restrictions have been evaluated for just one lens, as they may affect every single element in the lens array. First, with the aim of determining the valid range of DVL parameters to work with a real experimental setup, we propose here a resolution criterion. Using this approach, the minimum value the lens radius, $a$, can reach as a function of the fractal order has been evaluated. 

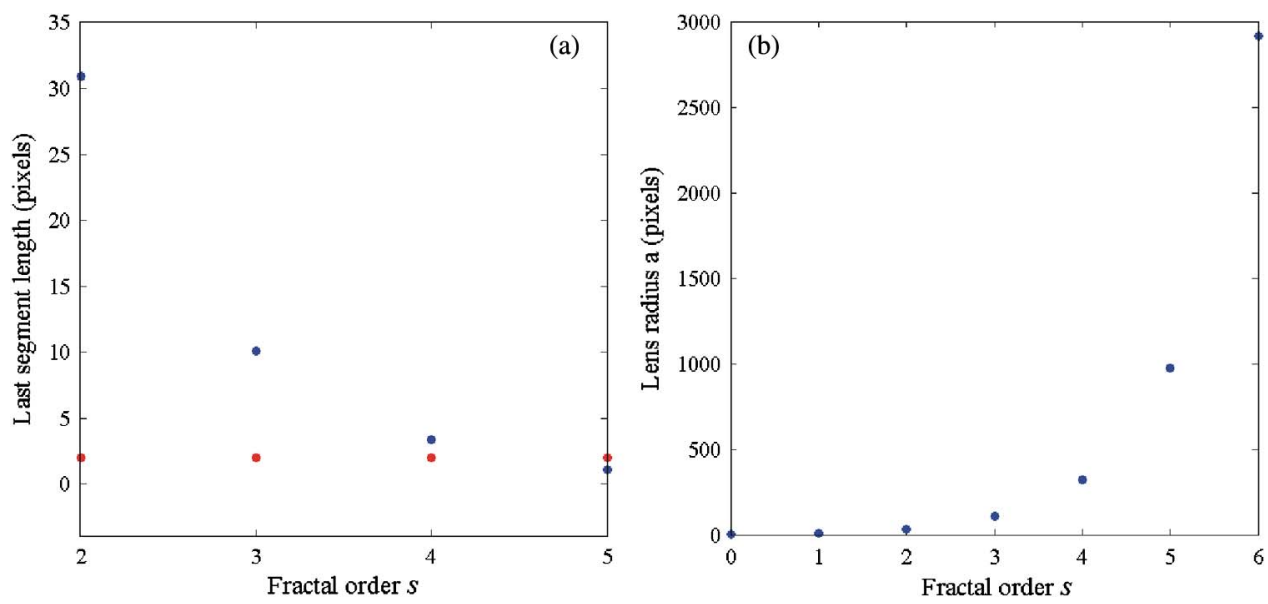

Fig. 8. Limits of spatial resolution for DVL design. (a) Last segment width as a function of the fractal order $s$ and minimum last segment width $L_{\min }$ (in red). (b) Minimum lens radius for a given fractal order $s$.

Our phase resolution criterion assumes that the last segment of the codified DVL must be at least two pixels wide, according to Nyquist sampling theorem. In this way, the $2 \pi$ phase variation can be properly codified. Notice now that the last segment begins in $r^{2} / a^{2}=1-3^{-s}$. Therefore its length in the real space $r$ is given by

$$
\mathrm{L}(s, a)=a\left[1-\left(1-3^{-s}\right)^{1 / 2}\right] .
$$

In Fig. 8(a) the last segment width as a function of lens fractal order is represented for the maximum lens radius allowed by the SLM (540 pixels). The minimum last segment width, $L_{\min }$, is also represented by the red points. The figure shows that for the maximum lens radius $a_{\max }=540$, the maximum fractal order is $s_{\max }=4$.

The minimum lens radius for a given fractal order $s$ can be expressed as
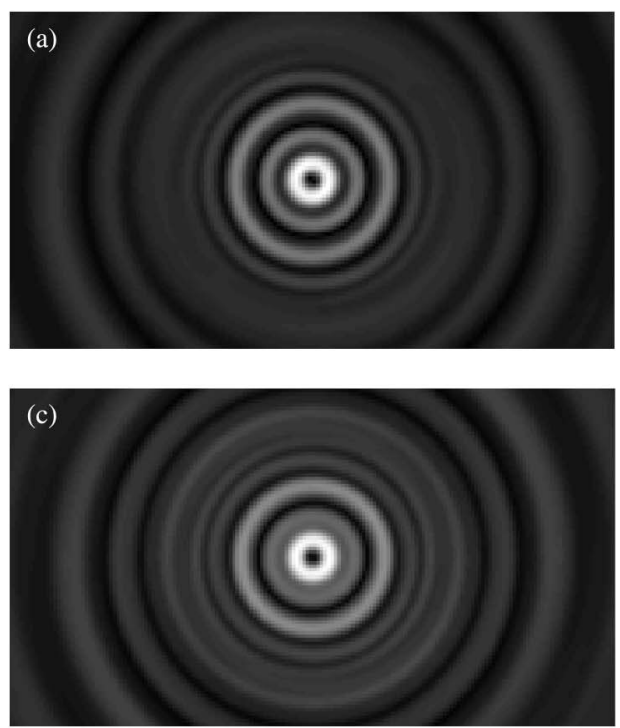

Fig. 9. Simulation of the irradiance at the main focus of a DVL with $s=2, m=2$ and diameter of (a) 269 pixels; (b) 259 pixels; (c) 249 pixels; and (d) 239 pixels, when the diaphragm used in the experiment has been considered.
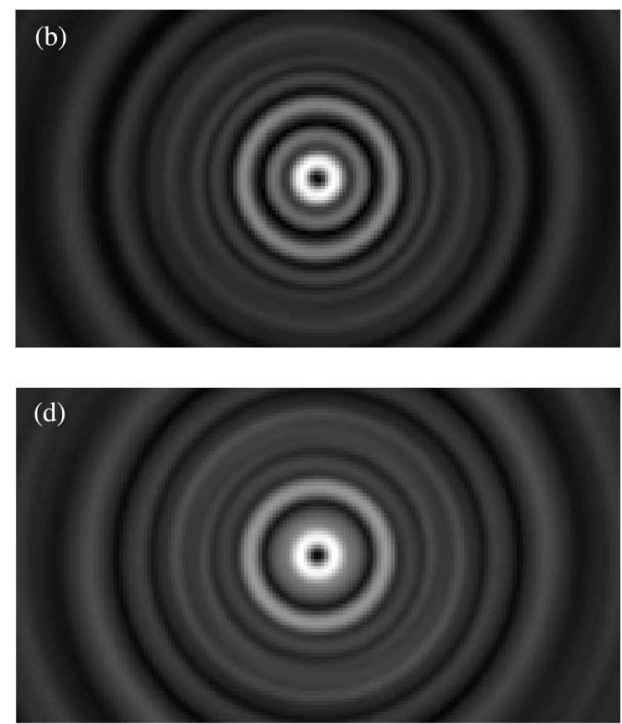

Equation 7 is represented in Fig. 8(b), showing how the lens minimum radius grows with fractal order when the proposed resolution criterion $\left(L_{\min }=2\right.$ pixels) is respected. Theoretically, for a DVL of fractal order $s=2$, the minimum possible radius fulfilling the resolution criterion is 35 pixels.

So far, only the last lens segment phase variation and low topological charge values have been considered. For very high topological charge values, the azimuthal phase presented by the DVLs around its center could vary so fast that it could not be correctly codified. Nevertheless, these cases have been disregarded as our study is restricted to low values of $m$.

Another factor that affects the spatial resolution of the lenses registered by the camera is the aperture of the diaphragm used in the telescopic system. Consider an object at the object focal point of a lens, 
with focal length $f$. In the image focal plane, spatial frequencies $\nu_{x}$ and $\nu_{y}$ are related to the real transversal distances $x$ and $y$ by the wavelength $\lambda$ and $f$ according to $[\underline{25}]$

$$
\nu_{x}=x / \lambda f \quad \nu_{y}=y / \lambda f .
$$

Now consider an iris diaphragm placed in the back focal plane of the lens. It will filter spatial frequencies in the Fourier plane depending on its aperture. We have experimentally determined the appropriate diaphragm aperture, accomplishing the trade-off between a good resolution and a limited noise coming from other diffraction orders, corresponding to a radius of $2 \mathrm{~mm}$.

Assuming that diaphragm aperture, a wavelength of $632.8 \mathrm{~nm}$ and a focal length of $200 \mathrm{~mm}$, the cutoff frequency or maximum spatial frequency passing through the diaphragm is about $1.6 \times 10^{4} \mathrm{~m}^{-1}$ or $15.8 \mathrm{lp} / \mathrm{mm}$.

To illustrate the effects of filtering, and considering the actual diaphragm aperture used in the experiment, simulated images of the irradiance pattern at the main focus are shown in Fig. 9. The cases of DVLs with fractal order $s=2$, topological charge $m=2$, and with a diameter ranging from 269 to 239 pixels are represented. It can be seen that for lenses with diameters shorter than 259 pixels, the irradiance pattern loses resolution and sharpness, and even the first rings of the lens seem to overlap.

\section{Conclusions}

The results reported in this paper represent a first approach to the generation of a 3D structure of vortices through a matrix of DVLs implemented on an SLM. Different sets of DVLs have been tested and characterized. The measured intensities show a good agreement with the simulations we have developed to contrast theory and experiment.

A theoretical estimation of the minimum dimension for a lens to be implemented with the sufficient resolution has been performed. The experimental minimum threshold for obtaining a correctly defined wave front, according to a given resolution criterion, has also been assessed.

We have obtained good results with lenses of 499 pixels (corresponding to about $2 \mathrm{~mm}$ in the plane where the lenses are imaged, after the telescopic system).

The angular velocity exhibited by the phase distribution produced by the array of DVLs has been visualized around the focal plane. Again, the agreement between theory and experiment is satisfactory.

Our experiments have proved the possibility of a simple design procedure of coupled DVLs with the desired range of topological charge. With our method, it is even possible to design arrays of spiral FraZPs with fractional topological charge [26] to break down the symmetry of the foci to produce anisotropic fractal vortex foci. As each individual DVL can be understood as a light gear capable of driving microstructures around its circumference [27], applications involving particle transfer and manipulation, where the pattern provided by the DVLs generates the adequate distribution of angular momentum, could be foreseen.

This work has been partly funded by the Government of Spain (projects FIS2010-15746, FIS2011-23175, and DPI2012-32994) and the Excellence Net from the Generalitat Valenciana about Medical Imaging (project ISIC/2012/013). Also funding from Generalitat Valenciana through Prometeo Excellence Programme (project PROMETEO/ 2012/021) and from Universitat Jaume I (project $\mathrm{P} 1 \cdot 1 \mathrm{~B} 2012-55)$ is acknowledged.

\section{References}

1. K. T. Gahagan and G. A. Swartzlander, Jr., "Optical vortex trapping of particles," Opt. Lett. 21, 827-829 (1996).

2. H. Me, M. E. J. Friese, N. R. Heckenberg, and H. RubinszteinDunlop, "Direct observation of transfer of angular momentum to absorptive particles from a laser beam with a phase singularity," Phys. Rev. Lett. 75, 826-829 (1995).

3. F. S. Roux, "Distribution of angular momentum and vortex morphology in optical beams," Opt. Commun. 242, 45-55 (2004).

4. G. Gbur and T. D. Visser, "Phase singularities and coherence vortices in linear optical systems," Opt. Commun. 259, 428-435 (2006).

5. W. M. Lee, X. C. Yuan, and W. C. Cheong, "Optical vortex beam shaping by use of highly efficient irregular spiral phase plates for optical micromanipulation," Opt. Lett. 29, 1796-1798 (2004).

6. G. Saavedra, W. D. Furlan, and J. A. Monsoriu, "Fractal zone plates," Opt. Lett. 28, 971-973 (2003).

7. S. H. Tao, X.-C. Yuan, J. Lin, and R. Burge, "Sequence of focused optical vortices generated by a spiral fractal zone plates," Appl. Phys. Lett. 89, 031105 (2006).

8. W. D. Furlan, F. Giménez, A. Calatayud, and J. A. Monsoriu, "Devil's vortex-lenses," Opt. Express 17, 21891-21896 (2009).

9. A. Calatayud, J. A. Rodrigo, L. Remón, W. D. Furlan, G. Cristóbal, and J. A. Monsoriu, "Experimental generation and characterization of Devil's vortex-lenses," Appl. Phys. B. 106, 915-919 (2012)

10. M. Mitry, D. C. Doughty, J. L. Chaloupka, and M. E. Anderson, "Experimental realization of the devil's vortex Fresnel lens with a programmable spatial light modulator," Appl. Opt. 51, 4103-4108 (2012).

11. S. Vyas and P. Senthikumaran, "Interferometric optical vortex array generador," Appl. Opt. 46, 2893-2898 (2007).

12. I. Moreno, J. A. Davis, D. M. Cottrell, N. Zhang, and X.-C. Yuan, "Encoding generalized phase functions on Dammann gratings," Opt. Lett. 35, 1536-1538 (2010).

13. P. García-Martínez, M. M. Sánchez-López, J. A. Davis, D. M. Cottrell, D. Sand, and I. Moreno, "Generation of Bessel beam arrays through Dammann gratings," Appl. Opt. 51, 1375-1381 (2012).

14. J. Yu, C. Zhou, W. Jia, W. Cao, J. Wu, and S. Wang, "Threedimensional Dammann vortex array with tunable topological charge," Appl. Opt. 51, 2485-2490 (2012).

15. S.-W. Cho, H. Kim, J. Hahn, and B. Lee, "Generation of multiple vortex-cones by direct phase modulation of annular aperture array," Appl. Opt. 51, 7295-7302 (2012).

16. J. Courtial, R. Zambrini, M. R. Dennis, and M. Vasnetsov, "Angular momentum of optical vortex arrays," Opt. Express 14, 938-949 (2006).

17. K. Ladavac and D. G. Grier, "Micromechanical pump assembled and driven by holographic optical vortices," Opt. Express 12, 1144-1149 (2004).

18. A. Mair, A. Vaziri, G. Weihs, and A. Zeilinger, "Entanglement of the orbital angular momentum states of photon," Nature 412, 313-316 (2001). 
19. S. H. Tao, B. C. Yang, H. Xia, and W. X. Yu, "Tailorable three-dimensional distribution of laser foci based on customized fractal zone plates," Laser Phys. Lett. 10, 035003 (2013).

20. B. Ma, B. Yao, Z. Li, M. Lei, S. Yan, P. Gao, D. Dan, and T. Ye, "Generation of three-dimensional optical structures by dynamic holograms displayed on a twisted nematic liquid crystal display," Appl. Phys. B 110, 531-537 (2013).

21. M. Reicherter, T. Haist, E. U. Wagemann, and H. J. Tiziani, "Optical particle trapping with computer-generated holograms written on a liquid-crystal display," Opt. Lett. 24, 608-610 (1999).
22. J. A. Monsoriu, W. D. Furlan, G. Saavedra, and F. Giménez, "Devil's lenses," Opt. Express 15, 13858-13864 (2007).

23. D. R. Chalice, "A characterization of the cantor function," Amer. Math. Monthly 98, 255-258 (1991).

24. W. D. Furlan, F. Giménez, A. Calatayud, L. Remón, and J. A. Monsoriu, "Volumetric multiple optical traps produced by devil's lenses," J. Eur. Opt. Soc. 5, 100375 (2010).

25. J. W. Goodman, Introduction to Fourier Optics, (McGraw-Hill, 1996).

26. L. Wei, Y. Gao, X. Wen, Z. Zhao, L. Cao, and Y. Gu, "Fractional spiral zone plates," J. Opt. Soc. Am. A 30, 233-237 (2013).

27. D. G. Grier, "A revolution in optical manipulation," Nature 424, 810-816 (2003). 